\title{
Enfermería Familiar y Comunitaria: mitos y falsas creencias de la
} reproducción asistida

\section{Family and Community Nursing: myths and false beliefs of artificial reproduction}

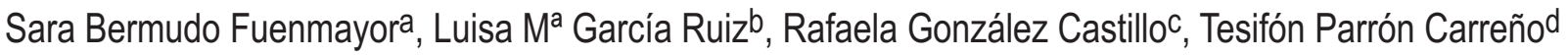 \\ a Unidad de Tocoginecología, Hospital de Poniente, España \\ b Unidad Pediátrica, Hospital Torrecárdenas, España \\ c Clínica IVI RMA Almería, España \\ d Servicio de la Delegación Territorial de Igualdad, Salud y Políticas Sociales, España
}

\section{Resumen}

Introducción: La reproducción es un proceso biológico que puede verse alterado por diversos motivos, como el aumento de la edad maternal para planificar la gestación, la aparición de nuevos conceptos de familia o pacientes que han pasado por un tratamiento de quimioterapia, reduciéndose la probabilidad de conseguir una gestación espontánea. Objetivos: Identificar los principales consejos sobre alimentación y ejercicio en el embarazo tras un procedimiento de reproducción asistida. Metodología: Se ha llevado a cabo una búsqueda bibliográfica en el año 2019, en las principales bases de datos Medline, ÍnDICEs-CSIC y CUIDEN. Resultados: Como profesionales sanitarios es nuestro deber la resolución de dudas, y corrección de posibles mitos o falsas creencias, tanto en la alimentación como en la práctica de ejercicio físico. Conclusiones: Para asegurar una atención integral de calidad, la mujer necesita de un equipo multidisciplinar coordinado trabajando en la misma dirección.

Palabras Clave: Enfermería; Atención Primaria de Salud; Reproducción Asistida; Alimentación; Ejercicio físico.

\begin{abstract}
Introduction: Reproduction is a biological process that can be modified for various reasons, such as the increase in maternal age for pregnancy planning, the appearance of new family concepts or patients who have undergone a chemotherapy treatment, reducing the probability of getting a spontaneous pregnancy. Objectives: Identify the main advice on diet and exercise in pregnancy after an assisted reproduction procedure. Methodology: A bibliographic review of articles in the Medline, ÍnDICEs-CSIC and CUIDEN databases was carried out in the year 2019. Results: As health professionals it is our duty to resolve doubts, and correct possible myths or false beliefs, both in food and in the practice of physical exercise. Conclusions: To guarantee comprehensive quality care, women need a coordinated multidisciplinary team working in the same direction.
\end{abstract}

Keywords: Nursing; Primary health care; Assisted reproduction; Feeding; Physical exercise. 


\section{Introducción}

$A^{10}$ lo largo de la vida, las personas pasan por diferentes etapas desde el nacimiento hasta su muerte. Durante todo el proceso de salud y enfermedad, son múltiples los profesionales que intervienen en algún momento, ya sea para conseguir un nivel óptimo de salud, a través de la promoción de la salud, prevención de la enfermedad, el diagnóstico, el tratamiento precoz, así como la rehabilitación tras un proceso agudo (Casado, 2016).

Dos de los profesionales sanitarios que van a acompañar a las personas desde que nacen hasta que mueren son los profesionales de Medicina y Enfermería Familiar y Comunitaria, abordando cada una de sus etapas, y poniendo énfasis a las actividades de prevención y promoción de la salud. Por su parte, la ginecóloga y la enfermera obstétrico-ginecológica (matrona), se especializan en la vida reproductiva de la mujer, desde la pubertad hasta la menopausia, pasando pues por la etapa de embarazo, parto y posparto. Juntos, dentro del equipo multidisciplinar de Atención Primaria, se marcarán como objetivo conseguir y mantener un nivel adecuado de salud sexual y reproductiva en la mujer, desde una visión integral de la mujer en su etapa reproductiva.

Pero, ¿qué pasa cuando el embarazo deriva de un tratamiento de reproducción asistida? La reproducción humana es un proceso biológico natural que, en ocasiones, puede verse alterado por diversos motivos, reduciéndose la probabilidad de conseguir una gestación espontánea (Mendiola, Ten, Vivero, \& Roca, 2005). Aunque los motivos suelen ser muy dispares, algunas de las causas pueden ser: la ausencia de producción de espermatozoides, la alteración de su número, movilidad y morfología, las alteraciones en la erección y/o la eyaculación, la anovulación femenina (por ejemplo en el síndrome del ovario poliquístico), las alteraciones morfológicas en el aparato reproductor femenino que impiden la fecundación o la implantación, etc. (Sociedad Española de Fertilidad, 2012).

Cuando nos encontramos ante algunas de las dificultades para la gestación espontánea, debemos estudiar y valorar, junto a la familia, el abordaje terapéutico que mejor se ajuste a sus necesidades. Debemos tener en cuenta siempre los deseos y las preferencias de la gestante y la familia, seleccionando el abordaje terapéutico más idóneo para cada paciente mediante la valoración de sus ventajas e inconvenientes, en términos de eficacia y seguridad (Brugo Omedo, Chillik, \& Kopelman, 2003; Fertilidad, 2012).
Hoy en día, la incorporación de la tecnología a la reproducción ha brindado una oportunidad de estudiar los procesos reproductivos básicos, y entre los métodos de reproducción asistida más destacados podemos encontrar: La Inseminación Artificial, que consiste en la colocación de una muestra de semen de un donante anónimo o de la pareja en el interior del útero de la mujer; la Fecundación in Vitro (FIV), que es la unión del óvulo con el espermatozoide en el laboratorio -tratamiento in vitro- con el fin de obtener embriones fecundados y transferirlos al útero materno; y la Ovodonación es una Fecundación in Vitro, con la diferencia, que el óvulo que se fecunda procede de una donante, no de la mujer que desea tener un hijo (Gual, 2011; «IVI RMA», s. f.; Solís, 2000).

Dicho esto, debemos plantearnos la siguiente pregunta: ¿existe diferencia entre un embarazo fisiológico y un embarazo tras un tratamiento de fertilidad? Cuando nos introducimos en el desarrollo fisiológico habitual de la vida fértil de la pareja que desea tener hijos, y detectamos algún problema de esterilidad, fertilidad, nos damos cuenta de que en pleno siglo XXI, este continúa siendo un tema tabú por la sociedad (Sociedad Española de Fertilidad, 2012).

La introducción de la mujer en el mundo laboral y la precariedad de los contratos que mantiene a la mayoría de las personas de un lado para otro hasta alcanzar cierta estabilidad están contribuyendo a que la mujer decida posponer el deseo de ser madre hasta una edad más tardía que hace años (Gual, 2011).

Si partimos de que la mujer tiene su pico de edad fértil entre los 23 y los 31 años, vemos que, a partir de esa edad, la probabilidad de conseguir un embarazo comienza a disminuir de forma progresiva, siendo la probabilidad de un $20 \%$ a partir de los 30 años, un $5 \%$ a partir de los 40 años y un 1\% a partir de los 35 años (Sinclair, Lea, Rees, \& Young, 2007).

Cada vez es más frecuente encontramos mayor diversidad en la demanda de reproducción asistida, desde parejas homosexuales, madres solteras con deseo gestacional, pacientes que han pasado por un tratamiento de quimioterapia y desean ser madres en un futuro, o hasta parejas que no se planteaban volver a tener hijos.

Actualmente, el concepto de Reproducción Asistida relacionado con Enfermedad comienza a alejarse de esta visión, puesto que el proceso de desarrollo del embrión, tanto en un embarazo natural como tras la transferencia embrionaria, no presentan diferencias, al igual que el desarrollo del bebé ("Clínica Eugin», s. f.). Otras de las principales preocupaciones de la población, son el aumento de enfermedades, abortos o malformaciones asociadas al tratamiento de reproducción asistida. 
Hoy en día, con la introducción del nuevo campo llamado epigenética, la creencia de que los genes es lo único que se transmite de padres a hijos está cambiando. La epigenética es considerada como la evolución de la teoría de la evolución, gracias a la cual podemos saber que aunque los genes se heredan por transmisión genética, es el medio ambiente el que actúa sobre los individuos, resultando diferentes manifestaciones genéticas (fenotipos) (Heijmans et al., 2008).

Heijmans etal (2008) es su estudio sobre la epigenética nos narra que en 1944 hubo en Holanda una época de hambruna, a final de la segunda guerra mundial, en la que la ocupación nazi bloqueó la llegada de alimentos a una población de los Países Bajos, obligándola a sobrevivir con apenas 1000 calorías al día. Los hijos de las mujeres embarazadas durante la hambruna mostraron cambios claros en su expresión genética 60 años después, derivando en mayores tasas de enfermedad coronaria, hipertensión, diabetes, esquizofrenia y obesidad. El efecto era mucho más marcado en los adultos cuyas madres habían sido expuestos a la hambruna en la primera parte del embarazo, donde se empieza a formar el nuevo ADN y las marcas epigenéticas. Además, ciertos problemas de salud se reflejaban también en sus hijos, es decir, los nietos de las mujeres que sufrieron la hambruna (Srisertpol, Tantrairatn, Tragrunwong, \& Khomphis, 2011).

Estas evidencias, junto al conocimiento de la influencia del ambiente entre otras, tienen un papel muy importante en la concienciación de la población en materia de nutrición antes y durante la gestación. Los primeros años de vida del ser humano son muy importantes para el desarrollo molecular, genético y de su metabolismo, con especial importancia en la alimentación por su impacto en la interacción epigenética comentada (Sánchez, Andeyro Garcia, \& Vázquez, 2015).

Cuando el cuerpo siente una amenaza, bien sea por estrés, falta de descanso, dietas bajas en calorías o niveles bajos de grasa corporal, existe una mayor probabilidad de que haya cierta dificultad para conseguir un embarazo (Spielmann, 1989). Además, en la fecundación tiene tanta importancia la capacidad reproductiva de la mujer como la del hombre, destacando que la calidad del esperma ha empeorado en las últimas décadas, con índices de disminución del nivel de testosterona (Elisabeth Carlsen, Aleksander Giwercman, Niels Keiding, 1992).

Por tanto, podemos afirmar que los cuidados $y$ el abordaje de un embarazo tras un tratamiento de reproducción asistida, seguirá las mismas pautas y consejos que daríamos a una mujer sana tras un embarazo espontáneo o natural. Por todo ello, el principal objetivo que nos proponemos con el presente trabajo es el de identificar, desde la perspectiva de la Enfermera de Atención Primaria, los principales consejos sobre alimentación y ejercicio en el embarazo tras un procedimiento de reproducción asistida, analizando la posible existencia de mitos y falsas creencias.

\section{Metodología}

Se realizó una revisión de artículos en la base de datos internacional Medline, ÍnDICEs-CSIC y CUIDEN en el año 2019, utilizando los términos DeCS: "Enfermería", "Atención Primaria de Salud", "Alimentación" y "Nutrición" en español, y "Nursing", "Primary Health Care" y "Nutrition" en inglés, y el término libre "Reproducción asistida", "Artificial Reproduction", en español e inglés, con el uso de los operadores AND y OR.

Tras definir los criterios de inclusión y exclusión, se realiza la selección y posterior análisis de los artículos que cumplan nuestros criterios propuestos, teniendo en cuenta la calidad del contenido de los mismos, y se interpretan los resultados.

Se evaluaron diferentes artículos en revistas científicas, revisiones bibliográficas y libros relacionados con la nuestra temática de estudio. Como limitadores de búsqueda, seleccionamos los artículos publicados desde el año 2000 hasta la fecha, quedan excluidos los publicados anteriormente.

Una vez realizamos la búsqueda bibliográfica con la estrategia de búsqueda establecida, y aplicados los filtros de temporalidad, procedemos a la lectura del resumen del resultado de nuestra búsqueda, seleccionando 78 artículos. Finalmente, teniendo en cuenta los criterios de inclusión y exclusión pautados, valorando el nivel de evidencia y calidad de los mismos, nos quedamos con 35 artículos.

\section{Resultados}

\section{Alimentación antes del embarazo}

Una alimentación sana y equilibrada, asegurando un adecuado aporte de vitaminas y minerales, siempre es recomendable, pero esta recomendación se vuelve necesidad cuando planeamos la búsqueda de un embarazo, con el objetivo de prevenir posibles complicaciones durante el período de gestación.

La eliminación de hábitos no saludables, como es el abandono del tabaco o del consumo de alcohol, también es importante en la prevención de malformaciones en el feto, entre otras patologías asociadas. Además, debemos asegurar un adecuado aporte de vitamina $D$ (presentes en lácteos, huevo y pescados azules), ácido fólico (presentes en verduras y hortalizas), vitamina B12 (presente en 
alimentos de origen animal) y hierro (en mariscos como las almejas), pero siempre con moderación, apostando por su consumo sin procesar, lo más natural posible (Sánchez et al., 2015).

Para la creación de una vida se necesita gran cantidad de energía, pero no se trata de "comer por dos" como muchas mujeres suelen decir. Si partimos de una buena alimentación de base, no es necesario suplementar la dieta diaria por encima de las 100-200 calorías extra en el primer trimestre, y las 300-400 calorías de más en los siguientes. Debemos destacar que el global de alimentos ingeridos, así como el total de calorías ingeridas diarias, cobran una mayor importancia que la de consumo de macronutrientes específicos. No obstante, no hay que olvidar que la dieta humana varía ampliamente según zonas geográficas (Sánchez et al., 2015).

Evitar la ingesta de carbohidratos durante el embarazo no es una buena opción, puesto que existen estudios que demuestran que aquellas mujeres que han seguido dietas bajas en carbohidrato pueden generar falsos positivos en pruebas como la de diabetes gestacional, por lo que es preferible asegurar un aporte moderado y distribuirlo en varias tomas a lo largo del día (Sánchez et al., 2015).

Durante el embarazo, existe cierta resistencia fisiológica a la insulina por parte del cuerpo materno, con el objetivo de facilitar el aporte de nutrientes al feto, pero es algo fisiológico. Por ello, una mujer que sigue una dieta de bajo contenido de hidratos de carbono, y que se somete a una prueba con una carga de glucosa pura de golpe, es muy probable que dé resultados falsamente positivos. Para evitarlo, podríamos realizar una prueba de hemoglobina glicosilada $(\mathrm{Hb} 1 \mathrm{Ac})$, que mide los niveles de glucosa durante los últimos meses. Además, los profesionales sanitarios deben recomendar la no limitación en carbohidratos durante los 3-4 días previos a la prueba de tolerancia a la glucosa, insistiendo en el consumo de al menos $150 \mathrm{~g}$ de carbohidrato al día. El aporte de HC por tanto será al menos de un $30 \%$ de las calorías totales, priorizando los de menor densidad (Sánchez-Muniz, Gesteiro, Espárrago Rodilla, Rodríguez Bernal, \& Bastida, 2013).

En cuanto a las proteínas, las necesidades se ven aumentadas durante el embarazo para el desarrollo fetal, sin embargo, el organismo materno disminuye su capacidad de transformar el amoniaco en urea, acumulándose, por lo que sería recomendable no aumentar en exceso el consumo de proteínas, con el fin de evitar el efecto tóxico que supondría su acúmulo. Lo ideal sería un $15-20 \%$ de las calorías totales, lo normal de una dieta equilibrada (Kalhan, 2000).
Por su parte, las grasas naturales son fundamentales durante el embarazo. Entre ellas destacamos la lactancia materna, con alto contenido de grasa saturada y colesterol, de gran importancia para la nutrición del bebé. Por ello, el organismo materno aumenta durante la etapa del embarazo, la producción de colesterol, ya que, junto con el ácido graso DHA, son elementos clave en el desarrollo cerebral. Se recomienda optar por pescados y carne más grasos e incluir lácteos con grasa (no desnatados), yemas de huevo, y aguacates, constituyendo un 30\% de las calorías totales (Sánchez-Muniz et al., 2013).

Como regla general, deberíamos favorecer el consumo de comida real, evitando y los suplementos, tanto para mejorar la fertilidad como para asegurar un buen embarazo (Sánchez et al., 2015).

Además de los macronutrientes, existen una serie de micronutrientes que no pueden faltar en la dieta, como es el caso del DHA, que se trata de un ácido graso, tipo omega 3, fundamental en el desarrollo del cerebro del bebé, que podemos encontrar en el aceite del pescado (Innis, 2008).

Algunos estudios han demostrado que los hijos de madres que consumían más de $340 \mathrm{~g}$ de pescado a la semana mejoraban en el desempeño de diversas pruebas cognitivas frente a los que ingerían una menor cantidad de pescado (Helland et al., 2003). Además, el aumento de consumo de pescado en la madre favorece la disminución de aparición de depresión postparto, así como mejora el desarrollo neuronal de los hijos. Se recomienda también priorizar pescados con bajo contenido en mercurio, como salmón, sardinas, gambas o camarones (Hibbeln et al., 2007).

En esta misma línea, debemos hablar del folato, que juega un papel fundamental en la construcción del ADN y la expresión genética del bebé. Es importante diferenciar el ácido fólico del folato, que podemos encontrarlo de forma natural en los alimentos. El ácido fólico es una versión sintética del folato, cuyo exceso está asociado a múltiples trastornos como el asma, cáncer y resistencia a la insulina (Davison \& Birch, 2008). Los folatos naturales podemos encontrarlos en los vegetales como espárragos, brócoli, coliflor y espinacas (Sánchez et al., 2015).

En cuanto a las vitaminas, debemos destacar la vitamina A, muy importante en el desarrollo de la función visual que podemos encontrarla en el hígado (Gilbert, 2002). La vitamina $D$ es clave en el desarrollo del sistema musculoesquelético y el sistema inmunitario del feto, especialmente en el último trimestre. Además, reduce complicaciones maternas como la preeclampsia (Hollis, Johnson, Hulsey, Ebeling, \& Wagner, 2011; Hyppönen et 
al., 2014). Estas vitaminas podemos encontrarlas en los huevos y lácteos de calidad (Sánchez et al., 2015).

Por último, en el desarrollo del bebé es muy importante la presencia de yodo, que podemos encontrarlo en pescados, mariscos y algas. Aunque algunos especialistas recomiendan reducir la sal durante el embarazo, la realizad es que no existe evidencia de que ofrezca beneficios a las embarazadas, mientras que existen casos demostrados de hiponatremia neonatal por restricción de sodio o ingestas muy elevadas de líquidos. Por todo ello, la recomendacione general respecto al yodo o la sal de mesa es ni eliminar la sal ni beber más de los necesario (Jellema, Balt, Broeze, Scheele, \& Weijmer, 2009).

\section{Alimentación durante el embarazo}

Debido a la gran importancia que la sociedad le ha otorgado al jamón, hablaremos de él en primer lugar. El tema del jamón en la dieta durante del embarazo, es uno de los que más suelen preocupar. Actualmente, hay muchas embarazadas que reciben información contradictoria sobre si pueden comer o no jamón serrano durante la gestación (Baquero-artigao et al., 2013). Esta recomendación deriva de la posible presencia de toxoplasma gondii en el cerdo. Estudios recientes demuestran que la prevalencia de toxoplasma gondii en el cerdo ha disminuido de un $90 \%$ en los años 60 , hasta un $1 \%$ en la actualidad. El proceso de curación del jamón superior a los 14 meses, suele inactivar el parásito, aunque no se puede afirmar de manera absoluta, constituyendo ese $1 \%$ de prevalencia (Hohlfeld et al., 1994).

Actualmente, se sigue desaconsejando el consumo de embutidos y carnes curadas, siempre que no hayan sido previamente congelados durante al menos 24 horas. Sin embargo, estos rumores sobre la posibilidad de comer jamón en el embarazo, no están del todo infundados. En 2012, el Centro Tecnológico Andaluz del Sector Cárnico (TEICA) dio a conocer la existencia de su estudio sobre toxoplasma en el jamón curado y su posible consumo por las mujeres embarazadas. Los resultados preliminares parecían indicar que el tiempo de curación y el contenido de sal del jamón pueden hacer inviable al toxoplasma. Ante las buenas previsiones, presentó un acuerdo con la Sociedad Española de Ginecología y Obstetricia (SEGO) para elaborar en un futuro un protocolo unificado sobre toxoplasma en las mujeres embarazadas (Santamaría, 2015).

La Asociación Española de Pediatría, ha realizado una serie de recomendaciones basadas en una Guía de la Sociedad Española de Infectología Pediátrica destacando la importancia de congelar el jamón entre 24 y 48 horas, debido a que el parásito se destruye a temperaturas de $-20^{\circ} \mathrm{C}$ en 24 horas antes del consumo, o bien consumir carne cocinada y bien hecha a más de $66^{\circ} \mathrm{C}$, o carne o embutido congelados (Baquero-artigao et al., 2013). Además, desde la Sociedad Española de Ginecología y Obstetricia hace tiempo que se afirma que las embarazadas sí pueden comer jamón sin congelar "siempre cuando haya pasado la cadena de producción sanitaria contrastada". De ahí, de nuevo, la importancia del etiquetado y de los controles de calidad.

Otros de los productos que más preocupación generan durante el embarazo, son los ahumados o el sushi. Los alimentos, especialmente durante el embarazo, es preferible evitar comerlos sin cocinar. El problema radica en la posible presencia de parásitos como el anisaki, que se evita al congelar el alimento durante 48 horas previas a la ingesta (Spielmann, 1989).

En cuanto al queso, debemos decir que algunos pueden tener una bacteria llamada listeria, resistente a bajas temperaturas, por lo que no podemos salvar el problema con la congelación. La listeria puede pueden causar una enfermedad conocida como listeriosis, la cual puede provocar aborto espontáneo, muerte fetal o graves problemas de salud en el bebé. Para evitar contraer la listeriosis, se recomienda no consumir los quesos blandos, como el queso blanco fresco estilo mexicano, queso feta o brie. En este caso debemos guiarnos por el etiquetado de los quesos o los lácteos para asegurar el consumo, buscando indicadores de que la leche sea pasteurizada y el queso sea elaborado con ese tipo de leche o calentada por encima de los $75^{\circ} \mathrm{C}$ (Flores-Quijano \& Heller-Rouassant, 2016).

La fruta y la verdura, al tratarse de alimentos extraídos directamente de la naturaleza, pueden contener todo tipo de insectos y parásitos, en especial las que sabemos que no han sido procesadas (huertos naturales). Por ello, toda la verdura que se vaya a ingerir durante el embarazo debe ser lavada de forma concienzuda, además de cocinarlos bien antes de consumirlos (American College of Nurse-Midwives, 2010).

Además de los anteriormente comentados, existen otros alimentos que debemos moderar su consumo durante la gestación, como es el caso de las aceitunas, por su alto contenido en sal. De forma fisiológica aumenta la tensión arterial de la embarazada, por lo que controlar estos niveles de tensión es importante, controlando el consumo de sal. En el caso de las aceitunas con anchoas, este segundo elemento puede hacernos dudar sobre la posibilidad de su consumo de forma segura, 
164 // Bermudo Fuenmayor et al.

pero en este caso, están tratadas cuando se introducen en la aceituna, por lo que la precaución sería igualmente respecto el contenido en sal. Distinto es si hablamos de las anchoas, que al igual que los ahumados y algunos pescados pueden contener anisaki, necesitando congelación previa al consumo (Sandra Lucía Restrepo M., Lorena Patricia Mancilla L., Beatriz Elena Parra S., Luz Mariela Manjarrés C. Natalia Janeth Zapata L., Paula Andrea Restrepo Ochoa., 2010).

\section{Ejercicio y embarazo tras un tratamiento de reproducción asistida}

Hoy en día, el embarazo es tratado por la sociedad como una enfermedad, alejando a la mujer de posibles exposiciones y privándola de actividades fisiológicas como es el ejercicio físico. Cuando además existe cierta dificultad para conseguir ese embarazo, la actividad física se convierte en un tema comprometido y muchos médicos deciden apostar por la recomendación del reposo casi absoluto, por lo que la mujer asume que lo más importante de esta etapa será la inactividad y el descanso (Blehar et al., 2013).

Sin embargo, son muchos los beneficios que la evidencia ha encontrado sobre el ejercicio físico y el desarrollo del embarazo, incluso de cara el parto y el puerperio, resaltando la importancia de recomendar a las futuras madres como Programa de Salud Maternal en Atención Primaria o en los talleres y sesiones ofertados. Además, si estudiamos un poco más a fondo el efecto del reposo en el embarazo, vemos que ocurre algo similar que cuando se recomienda inmovilización para el tratamiento de una lesión, que no existe evidencia de beneficio alguno. Si remontamos a nuestros antepasados, podemos afirmar que nuestra especie no hubiese sobrevivido si el embarazo hubiese sido sinónimo de invalidez (Salvesen, Hem, \& Sundgot-Borgen, 2012).

Según varios estudios, durante la etapa de embarazo, el $90 \%$ de las mujeres sedentarias presentan una mayor tendencia a la diástasis abdominal (separación del músculo recto del abdomen), frente al $12,5 \%$ de las que sí continuaron practicando deporte (Chiarello, Falzone, McCaslin, Patel, \& Ulery, 2005).

El mantenimiento del peso corporal será otros de los beneficios del ejercicio físico durante el embarazo, evitando esos kilos de más. (Streuling et al., 2011) Otros beneficios serán la disminución del riesgo de padecer hipertensión arterial, diabetes gestacional, 0 preeclampsia. Otros estudios, han demostrado que las mujeres que continuaron practicando ejercicio durante el embarazo, tuvieron partos más cortos y menos problemáticos, con menor riesgo de sufrimiento fetal agudo (Zhang, Solomon, Manson, \& Hu, 2006).

Si buscamos indicadores positivos de la práctica deportiva de baja intensidad durante las primeras fases del embarazo, podemos destacar una reducción en el tiempo de hospitalización, la incidencia de cesáreas, y las pruebas de Apgar positivas (Hall \& Kaufmann, 1987). Otro tipo de entrenamiento, como es el de fuerza, reduce la necesidad de insulina en mujeres embarazadas diagnosticadas de diabetes gestacional (Brankston, Mitchell, Ryan, \& Okun, 2004).

Pero, al igual que con la alimentación, en el término medio está la virtud, por lo que hay que adaptar la intensidad del ejercicio a la capacidad de cada mujer, no siendo recomendado superar el $90 \%$ de la intensidad máxima (Salvesen et al., 2012).

Previamente hablábamos de la influencia de la nutrición y la epigenética en la madre y en el bebé y como el comportamiento de la madre marcará la expresión genética del bebé, por lo que, de igual modo, la actividad física de la madre, además de mejorar su nivel cardiovascular, mejora el corazón del bebé (Clapp, 1996).

Cuando hablamos de actividad física durante el embarazo, no quiere decir que la mujer vaya a inscribirse en una maratón o una clase de Crossfit, tampoco será el momento de incorporar o probar actividades que nunca se hayan llevado a cabo. Trabajar la movilidad, la postura y la fuerza, acompañado de un entrenamiento aeróbico aporta grandes beneficios para la madre y el bebé tanto en la etapa del embarazo, como en el momento del parto y posparto (Hall \& Kaufmann, 1987).

En el embarazo, es frecuente que la futura mamá refiera molestias o dolor de espalda, cuello, cabeza... Durante el embarazo el cuerpo experimenta grandes cambios fisiológicos con el fin de adaptar el cuerpo al desarrollo y crecimiento del bebé, pero el sufrimiento no forma parte de este proceso (Salvesen et al., 2012).

Cada mujer tiene una historia detrás que debemos conocer antes de pautar cualquier plan de entrenamiento, ejercicio o educación postural, pues no todo el mundo partirá del mismo punto. Una adecuada evaluación inicial nos permitirá conocer desde donde iniciamos y hasta dónde podríamos llegar. Como hemos comentado, no vamos a recomendar a una embaraza apuntarse a baloncesto si nunca antes lo ha practicado. Si proponemos ejercicio físico a una mujer que nunca ha realizado actividad alguna, es probable que el simple hecho de adaptar su cuerpo al aumento de peso, ya sea suficiente para desestabilizar la postura y obtener resultados para 
que estas disfunciones o dolencias aparezcan (Chacón Borrego, Ubago Jiménez, Guardia García, Padial Ruz, \& Cepero González, 2018).

Al corregir la postura, el dolor de espalda se corregirá poco a poco, así como las molestias de espalda y cervicales. Al reducir la presión sobre los abdominales, se minimiza el riesgo de producirse una diástasis abdominal, conservando la musculatura abdominal después del parto (Rodríguez-Matoso et al., 2012).

Otro aspecto importante es evitar el uso de tacones altos durante el embarazo, puesto que el tacón tiende a llevar el peso del cuerpo hacia los dedos, provocando una cascada de compensaciones hacia arriba: pelvis rotada, hiperextensión lumbar, cuello adelantado... alterando la postura corporal y favoreciendo la aparición de esas molestias o dolor que refieren las embarazadas (Silva \& Siqueira, 2013).

En cuanto al entrenamiento de fuerza, debemos resaltar sus grandes beneficios antes, durante y después del embarazo. Cuando hablamos de entrenamiento de fuerza, no tiene nada que ver con el culturismo o la imagen social que tenemos de que una mujer que realiza ejercicios de fuerza es poco femenina. La inclusión de 2-3 veces por semana de algún tipo de entrenamiento de fuerza sería ideal, con especial énfasis en los abdominales y el suelo pélvico (Chiarello et al., 2005).

La zona abdominal es una de las más dañadas tras la distensión fisiológica de la piel, los tejidos y los músculos, característica del embarazo. Al trabajar la musculatura del abdomen profundo, estaremos tratando de evitar la debilidad que se produce en esta zona durante y después del parto. Además, el abdomen es una parte muy importante de la estructura del suelo pélvico, responsable de la continencia vesical y de la acomodación de los órganos urogenitales. Unas disfunciones de algunas de las partes ocasionarán problemas como dispareunia, vaginismo, prolapsos, incontinencia urinaria o fecal... etc. (Pereira et al., 2012). El uso de pesas o el peso corporal para este tipo de entrenamiento, ayudará a estabilizar la espalda y trabajar el equilibrio (Piper TJ, 2012).

Cuando trabajamos el suelo pélvico, no debemos olvidar a nuestros antepasados, quienes tenían mayor número de hijos que en la actualidad, con menos problemas en su suelo pélvico, resultando la principal diferencia que se movían más. El informe conjunto de terminología y estandarización del suelo pélvico femenino elaborado por la International Urogynecological Association (IUGA) y el Institut Català de la Salut (ICS), remarca que los diagnósticos más frecuentes son aquellos para los que hay evidencia de una prevalencia de un $10 \%$ o más en mujeres que presentan síntomas de disfunción del suelo pélvico tras el embarazo (Observatorio Nacional de la Incontinencia, 2009).

A nivel internacional, la International Consultation on Urological Diseases (ICUD), estima que la prevalencia de la incontinencia urinaria, independientemente de su tipo o grado en mujeres de edad media o avanzada, oscila entre el 25 y el $45 \%$, porcentaje que aumenta con la edad (Milsom et al., 2013).

El aumento del tamaño del útero y su colocación conforme avanza la gestación a nivel abdominal, contribuyen a un debilitamiento de la musculatura del suelo pélvico. Esta debilidad empeora con la distensión final que ocurre durante el parto Todos estos cambios, sobre todo durante el último trimestre, pueden dar lugar a pequeñas alteraciones como pequeños escapes de orina ante esfuerzos (Cerrato-Lópeza et al., 2017).

No obstante, no debemos olvidar que sea gestación biológica o reproducción asistida, nos encontramos ante una situación novedosa para la homeostasis de la mujer, por lo que debemos ir adaptando cada sesión según la evolución del embarazo. A partir del segundo trimestre, debería evitarse la realización de ejercicios en posición supina, puesto que podría reducir el flujo sanguíneo hacia el útero. Además, no debemos olvidar a la espalda y los hombros, puesto que un entrenamiento global (full body) nos ayudará a evitar la deformación postural (RodríguezMatoso et al., 2012).

Durante el embarazo las articulaciones se vuelven más elásticas, y la barriga tiende a desplazar levemente el centro de gravedad, pudiendo verse afectados el equilibrio y la estabilidad, acentuado las precauciones al realizar ejercicios corporales (Chacón Borrego et al., 2018).

Para concluir, destacar que el hecho de estar embarazada es una suerte y un privilegio, y para nada un estado patológico. Por ello, todas nuestras actividades cotidianas debemos seguir manteniéndolas con normalidad. Si a la mujer le gusta correr, la única precaución que debes tomar es mantener una buena técnica para evitar lesiones, dado que el cuerpo va cambiando y no controlamos muy bien ni el peso (con repercusión en las articulaciones) ni las nuevas dimensiones. En el último trimestre, sí que sería recomendable el control de las pulsaciones por debajo de 140, evitando el ejercicio de alta intensidad. Pasando el primer trimestre, la incorporación de la bicicleta estática o la natación pueden colaborar en la reducción del impacto articular, siendo suficientes 2 sesiones semanales de unos 15-30 minutos, un poco más si partíamos de una buena base física (Hall \& Kaufmann, 1987). 
Con todo esto, no debemos hacer pensar que una mujer embarazada está en igualdad de condiciones que una que no lo esté, puesto que debe evitar riesgos innecesarios, dejando la innovación y la experimentación física para después del parto. Distinto es también el caso de presentar náuseas o fatiga, aconsejando acudir a un profesional que evalúe el punto de partida y juntos, poder programar los pasos a dar.

\section{Conclusiones}

En 1966, una de los principales referentes de la enfermería, Virginia Henderson, publicó en su libro The Nature of Nursing una definición para la enfermería como:

La función singular de la enfermería es asistir al individuo, enfermo o no, en la realización de esas actividades que contribuyen a su salud o su recuperación (o a una muerte placentera) y que él llevaría a cabo sin ayuda si tuviera la fuerza, la voluntad o el conocimiento necesarios. Y hacer esto de tal manera que le ayude a adquirir independencia lo más rápidamente posible (Henderson, 1994).

Esa definición ha sido adoptada por múltiples autores/ as, modelos y teorías enfermeras a lo largo de la historia (Bellido \& Lendínez, 2013).

Siguiendo la definición de Virginia Henderson, podemos afirmar que las principales funciones de la enfermería son asistenciales, docentes, investigadoras y gestoras, funciones que cobran una mayor importancia en el primer nivel asistencial, la base del sistema sanitario, la Atención Primaria.

Así mismo, cuando aparece un tema novedoso como es la reproducción asistida, los profesionales de la medicina y de la enfermería de atención primaria, como equipo multidisciplinar. Todos ellos deben marcarse como objetivo en materias preventivas y de promoción de la salud, conseguir y mantener un nivel adecuado de salud sexual y reproductiva en la mujer, desde una visión integral de la mujer en su etapa reproductiva, identificando los principales consejos sobre alimentación y ejercicio en el embarazo tras un procedimiento de reproducción asistida, analizando la posible existencia de mitos y falsas creencias.

Por todo ello, y a modo de conclusión en cuanto a alimentación nos referimos, deberíamos señalar la importancia de priorizar alimentos con alta densidad nutricional, alejándonos de dietas hipocalóricas y bajas en grasa, pero con énfasis en ciertos nutrientes especialmente relevantes para soportar una nueva vida, con especial importancia en la vigilancia del etiquetado de los alimentos prestando una mayor atención al jamón, las carnes procesadas y la leche que debe ser pasteurizada.
La eliminación de hábitos no saludables como es el abandono del tabaco o del consumo de alcohol, también es importante en la prevención de malformaciones en el feto, entre otras patologías asociadas.

En cuanto al ejercicio físico, cabe destacar que son múltiples los beneficios que la evidencia ha encontrado en su práctica, tanto el desarrollo del embarazo, como de cara al parto y el puerperio, resaltando la importancia de recomendar a las futuras madres Programas de Salud Maternal en Atención Primaria, así como talleres y sesiones ofertados. Entre los beneficios del ejercicio en el embarazo podemos identificar estudios que evidencian una menor tendencia a la diástasis abdominal, mantenimiento del peso corporal, o la facilitación de partos más cortos y menos problemáticos, con menor riesgo de sufrimiento fetal agudo. Trabajar la postura, la movilidad, el suelo pélvico y la fuerza, acompañado de un entrenamiento aeróbico, son pilares fundamentales en el desarrollo de un embarazo saludable, evitando la aparición de dolor neuromuscular, adopción de malas posturas o la sobrecarga de la zona lumbar.

Aunque aún nos queda mucho camino por recorrer, para asegurar una atención integral de calidad, la mujer necesita de un equipo multidisciplinar coordinado, donde profesionales como matronas y ginecólogos, médicos y enfermeros de atención primaria, se dan la mano para trabajar en la misma dirección, resolviendo dudas, aclarando y corrigiendo posibles errores, mitos y falsas y creencias y asegurando un embarazo saludable.

\section{Referencias Bibliográficas}

American College of Nurse-Midwives. (2010). Compártelo con mujeres. Alimentación saludable durante el embarazo. Journal of Midwifery \& Women's Health, 55(1), e23-e24. https://doi.org/10.1016/j. jmwh.2009.11.009

Baquero-artigao, F., Castillo, F., Fuentes, I., Goncé, A., Fortuny, C., Fernández-miranda, M., ... Ramos, J. (2013). Guía de la Sociedad Española de Infectología Pediátrica para el diagnóstico y tratamiento de la toxoplasmosis congénita. Asociación Española de Pediatría, 79(2), 116.e1-116.e16. https://doi. org/10.1016/j.anpedi.2012.12.001

Bellido, J., \& Lendínez, J. (2013). Proceso Enfermero desde el modelo de cuidados de Virginia Henderson (llustre Co). Jaén: Ilustre Colegio Oficial de Enfermería de Jaén. https://doi.org/J 1574-2010

Blehar, M. ., Spong, C., Grady, C., Goldkind, S., Sahin, L., \& Clayton, J. A. (2013). Enrolling Pregnant Women: Issues in Clinical Research. Womens Health, 1(23), e39-e45. 
Brankston, G. N., Mitchell, B. F., Ryan, E. A., \& Okun, N. B. (2004). Resistance exercise decreases the need for insulin in overweight women with gestational diabetes mellitus. American Journal of Obstetrics \& Gynecology, 190(1), 188-193. https://doi. org/10.1016/S0002-9378(03)00951-7

Brugo Omedo, S.; Chillik, C.; Kopelman, S. (2003). Definición y causas de infertilidad. Revista Colombiana de Obstetricia y Ginecología, 54(4), 227-248. Recuperado de http://www.redalyc.org/ articulo.oa?id=195214309003

Casado, M. (2016). Reproducción humana asistida: los problemas que suscita desde la bioética y el derecho. Papers. Revista de Sociologia, 53, 37. https://doi. org/10.5565/rev/papers.1893

Cerrato-Lópeza, C., Arroyo-Rodrígueza, P., CabreraMartosa, I., Torres-Sáncheza, I., Checa-Morenob, V., \& Valenza, M. C. (2017). Modificaciones musculoesqueléticas en mujeres embarazadas con dolor lumbopélvico. Fisioterapia, 39(1), 18-24.

Chacón Borrego, F., Ubago Jiménez, J., Guardia García, J., Padial Ruz, R., \& Cepero González, M. (2018). Educación e higiene postural en el ámbito de la Educación Física: papel del maestro en la prevención de lesiones: revisión sistemática. Retos, 34, 8-13.

Chiarello, C., Falzone, L., McCaslin, K., Patel, M., \& Ulery, K. (2005). The Effects of an Exercise Program on Diastasis Recti Abdominis. Journal of Women's Health Physical Therapy, 29(1), 11-16.

ClappIII,J.F.(1996). Morphometricand neurodevelopmental outcome at age five years of the offspring of women who continued to exercise regularly throughout pregnancy. The Journal of Pediatrics, 129(6), 856-863. https:// doi.org/10.1016/S0022-3476(96)70029-X

Clínica Eugin. (s. f.). Clínica Eugin. Recuperado de https:// www.eugin.es/preguntas/embarazo-reproduccionasistidal

Davison, K. K., \& Birch, L. L. (2008). NIH Public Access, 64(12), 2391-2404. https://doi.org/10.1038/jid.2014.371

Elisabeth Carlsen, Aleksander Giwercman, Niels Keiding, N. E. S. (1992). Evidence for decreasing quality of semen during past 50 years. British Medical Journal, 305, 609-613. https://doi.org/10.1136/ bmj.305.6854.609

Flores-Quijano, M., \& Heller-Rouassant, S. (2016). Embarazo y Lactancia. Gaceta Médica de México, 152(1), 6-12.
Gilbert, T. (2002). Retinoids and the Kidney Vitamin A and kidney development. Nephrology Dialysis Transplantation, 17(9), 78-80. https://doi.org/10.1093/ ndt/17.suppl_9.78

Gual,A.M.(2011).Maternidadytécnicasdereproducción asistida:un análisis, desde la perspectiva de género, de los conflictos y experiencias de las mujeres usuarias. Recuperado de https://www. tesisenred.net/handle/10803/32098\#page=1

Hall, D. C., \& Kaufmann, D. A. (1987). Effects of aerobic and strength conditioning on pregnancy outcomes. American journal of obstetrics and gynecology, 157(5), 1199-1203. https://doi.org/10.1016/s00029378(87)80294-6

Heijmans, B. T., Tobi, E. W., Stein, A. D., Putter, H., Blauw, G. J., Susser, E. S., ... Lumey, L. H. (2008). Persistent epigenetic differences associated with prenatal exposure to famine in humans. Proceedings of the National Academy of Sciences, 105(44), 1704617049. https://doi.org/10.1073/pnas.0806560105

Helland, I. B., Smith, L., Saarem, K., Saugstad, O. D., Drevon, C. A., \& Docosahexaenoic, A. O. (2003). Pregnancy and Lactation Augments Children 's IQ at 4 Years of Age. Pediatrics, 111(1), 39-44. https://doi. org/10.1542/peds.111.1.e39

Henderson, V. (1994). Desarrollo de un concepto personal. En: Naturaleza de la enfermería, reflexiones 25 años después. México: E. I. McGraw-Hill.

Hibbeln, J. R., Davis, J. M., Steer, C., Emmett, P., Rogers, I., Williams, C., \& Golding, J. (2007). Maternal seafood consumption in pregnancy and neurodevelopmental outcomes in childhood (ALSPAC study): an observational cohort study. Lancet, 369(9561), 578585. https://doi.org/10.1016/S0140-6736(07)60277-3

Hohlfeld, P., Daffos, F., Costa, J., Thulliez, P., Forestier, F., \& Vidaud, M. (1994). Prenatal diagnosis of congenital toxoplasmosis with a polymerase-chain. Reaction test on amniotic fluid. The New England Journal of Medicine, 331(11), 695-699.

Hollis, B. W., Johnson, D., Hulsey, T. C., Ebeling, M., \& Wagner, C. L. (2011). Vitamin D supplementation during pregnancy: Double-blind, randomized clinical trial of safety and effectiveness. Journal of Bone and Mineral Research, 26(10), 2341-2357. https:// doi.org/10.1002/jbmr.463

Hyppönen, E., Cavadino, A., Williams, D., Fraser, A., Vereczkey, A., Fraser, W. D., ... Czeizel, A. E. 
(2014). Vitamin D and Pre-Eclampsia: Original data, systematic review and meta-analysis. Annals of Nutrition and Metabolism, 63(4), 331-340. https:// doi.org/10.1159/000358338

Innis, S. M. (2008). Dietary omega 3 fatty acids and the developing brain. Brain Research, 1237, 35-43. https://doi.org/10.1016/J.BRAINRES.2008.08.078

IVI RMA. (s. f.). Recuperado de www.ivi.es

Jellema, J., Balt, J., Broeze, K., Scheele, F., \& Weijmer, M. (2009). Hyponatraemia during pregnancy. The Internet Journal of Gynecology and Obstetrics, 12(1), 1-6. https://doi.org/10.5580/ebe

Kalhan, S. C. (2000). Protein metabolism in pregnancy. American Journal of Clinical Nutrition, 71(5 Suppl), 1249S-1255S. https://doi.org/10.1016/ S0950-351X(96)80726-9

Mendiola J, Ten J, Vivero G, Roca M, B. R. (2005). Esterilidad y Reproducción Asistida: Una perspectiva histórica. Revista iberoamericana de fertilidad, 22(1), 15-22. Recuperado de http://www. revistafertilidad.org/RecursosWEB/fertilidad/Ferti-EnFeb05-Trabaj01.pdf

Milsom, I., Altman, D., Cartwright, R., Lapitan, M., Nelson, R., Sillén, U., \& Tikkinen, K. (2013). Epidemiology of Urinary Incontinence (UI) and other Lower Urinary Tract Symptoms (LUTS), Pelvic Organ Prolapse (POP) and Anal Incontinence (AL). En Institute of Clinical Sciences (pp. 15-107). Paris: ICUD-EAU.

Observatorio Nacional de la Incontinencia. (2009). Percepción del Paciente con Incontinencia Urinaria (I.U.). Recuperado de http://www. observatoriodelaincontinencia.es/pdf/Percepcion_ Paciente_IU.pdf

Pereira, L. C., Botelho, S., Marques, J., Amorim, C. F., Lanza, A. H., Palma, P., \& Riccetto, C. (2012). Are transversus abdominis/oblique internal and pelvic floor muscles coactivated during pregnancy and postpartum? Neurourology and Urodynamics, 32(5), 416-419. https://doi.org/doi:10.1002/nau.22315

Piper TJ. (2012). Core training exercise selection during pregnancy strength and conditioning journal. Medicine \& Science in Sports \& Exercise, 34(1), 1-5.

Restrepo, S.L., Mancilla, L. P., Parra, B. E., Manjarrés, L. M., Zapata, N.J., Ochoa, P. A. R., \& Martínez, M.I. (2010). Evaluación del estado nutricional y de los cuidados ante patologías durante la gestación, en embarazadas que asisten a sus controles prenatales entre agosto y septiembre de 2016. Revista Chilena de Nutrición, 37(1), 18-30. https://doi.org/http:// dx.doi.org/10.4067/S0717-75182010000100002

Rodríguez-Matoso, D., García-Manso, J. M., Sarmiento, S., de Saa, Y., Vaamonde, D., Rodríguez-Ruiz, D., \& da Silva-Grigoletto, M. E. (2012). Prescripción del ejercicio físico durante el embarazo. Revista Andaluza de Medicina del Deporte, 5(1), 28-40. https://doi.org/10.1016/S1888-7546(12)70006-0

Salvesen, K. Å., Hem, E., \& Sundgot-Borgen, J. (2012). Fetal wellbeing may be compromised during strenuous exercise among pregnant elite athletes. British Journal of Sports Medicine, 46(4), 279-283. Recuperado de http://bjsm.bmj.com/ content/46/4/279.abstract

Sánchez-Muniz, F. J., Gesteiro, E., Espárrago Rodilla, M., Rodríguez Bernal, B., \& Bastida, S. (2013). La alimentación de la madre durante el embarazo condiciona el desarrollo pancreático, el estatus hormonal del feto y la concentración de biomarcadores al nacimiento de diabetes mellitus y síndrome metabólico. Nutricion Hospitalaria, 28(2), 250-274. https://doi.org/10.3305/nh.2013.28.2.6307

Sánchez, Á., Andeyro Garcia, M., \& Vázquez, C. (2015). Guía de alimentación para embarazadas. Mi bebé y yo. Madrid: Medicadiet. Recuperado de http:// www.madrid.org/cs/Satellite?blobcol=urldata\&blobhe ader=application/pdf\&blobheadername $1=$ Content-di sposition\&blobheadername $2=$ cadena\&blobheaderv alue $1=$ filename $=$ Guia-Alimentacion-EmbazaradasMedicadiet[1].pdf\&blobheadervalue2=language=es\& site=HospitalGr

Santamaría, F. (2015). Actualización Toxoplasmosis. Granada. Recuperado de http://www.hvn.es/ servicios_asistenciales/ginecologia_y_obstetricia/ ficheros/actividad_docente_e_investigadoral clases_residentes/2015/clase2015_actualizacion_ toxoplasmosis.pdf

Silva, A. M., \& Siqueira, G. R. (2013). Implications of high-heeled shoes on body posture of adolescents. Revista Paulista de Pediatria, 31(2), 265-271.

Sinclair, K., Lea, R., Rees, W., \& Young, L. (2007). The developmental origins of health and disease: current theories and epigenetic mechanisms. Reproduction in Domestic Ruminants, 6(1), 425-443. https://doi. org/10.5661/RDR-VI-425 
Sociedad Española de Fertilidad, S. (2012). Saber más sobre fertilidad y reproducción asistida. Ministerio de Sanidad, Servicios Sociales e Igualdad (Vol. 1). Recuperado de https://www.sefertilidad.net/ docs/pacientes/spr_sef_fertilidad.pdf

Solís, L. S. (2000). Tecnicas de reproduccion asistida. Aspectos bioeticos. Cuadernos de Bioética, 1, 3747.

Spielmann, K. A. (1989). A review: Dietary restrictions on hunter-gatherer women and the implications for fertility and infant mortality. Human Ecology, 17(3), 321-345. https://doi.org/10.1007/BF00889022

Srisertpol, J., Tantrairatn, S., Tragrunwong, P., \& Khomphis, V. (2011). Estimation of the mathematical model of the reheating furnace walking hearth type in heating curve up process. International Journal of Mathematical Models and Methods in Applied Sciences, 5(1), 167-174. https://doi.org/10.1111/ j.1471-0528.2008.01822.x

Streuling, I., Beyerlein, A., Rosenfeld, E., Hofmann, H., Schulz, T., \& Kries, R. von. (2011). Physical activity and gestational weight gain: a meta-analysis of intervention trials. An International Journal of Obstetrics \& Gynaecology, 118(3), 278-284. https://doi.org/10.1111/j.1471-0528.2010.02801.x

Zhang, C., Solomon, C., Manson, J., \& Hu, F. (2006). A prospective study of pregravid physical activity and sedentary behaviors in relation to the risk for gestational diabetes mellitus. Archives of Internal Medicine, 166(5), 543-548. http://dx.doi. org/10.1001/archinte.166.5.543 